\title{
HIM: Hand Gesture Recognition in Mobile-Learning
}

\author{
Nitin Sharma \\ Lovely Professional University, Phagwara, Punjab, \\ India
}

\author{
Harsh Sharma \\ Lovely Professional University, Phagwara, Punjab, \\ India
}

\begin{abstract}
Mobile learning is the technology which makes many people around the world to learn by sitting at their home. So by considering the importance of the mobile learning the aim of this research is to build a system which makes the dumb people to learn and communicate with other people in the language which they know (hand sign language) and also the people who want to communicate with others without touching the keyboard or the mobile they can use their hand movements for such purpose. To achieve this, the research will implement the hand gesture recognition in mobile device which includes laptops, mobile phones, tablet pc. The methodology of this research involves the capturing video through camera, segmentation of the hand information from captured video, real time tracking and recognition of hand gesture, and store the gestures in database along with the text, when dumb people or the other people wants to communicate with each other the camera will capture their hand movements and match these movements in database, if match occurs then it will send the corresponding text to the people either in speech or text. If it does not match then the user can add this new gesture of the hand in database along with the text which will be enter by the user. This text will corresponds to the text form of that particular gesture of the hand.
\end{abstract}

\section{Keywords}

Database, Hand gesture recognition, Mobile Learning, Real time tracking, Segmentation, Speech or Text, Video.

\section{INTRODUCTION}

In the today's world with the development of the technology in our lives the work is becoming more easy for the people and the more and more people attracted towards the new technologies for e.g. as we have the computers in our home five years ago but when the laptops came in more and more people have bough the laptops and computer become more outdated in the same way we have the landline phones before when the mobile phones was not there in the market and when the mobile phones came in it totally replaces the landline phones and now all the people have the mobile phones at their home. The development of these technologies also has a greater effect on the education too. As the mobile devices became a crucial part of our life it actually helps us in the educational field as well through mobile learning. The mobile learning means the learning through the mobile devices so we can say that the mobile leaning is the anywhere and anytime leaning. It allows the people from all over the world to connect with each other through the mobile devices and can communicate with each other by using the internet via their mobile devices. With the help of mobile learning people around the world can share their problem with each other .Mobile learning is helpful at those places where the people do not have the enough money for the infrastructure of the school and the colleges at those places the mobile learning is really helpful because of the many reasons the first one is that the mobile learning is much more cheaper than that of the traditional way of class room learning because the mobile phones are much more cheaper than the infrastructure of the colleges and the schools and many people can afford the mobile devices now days. It allows the people to connect to the people all around the world and can enhance their knowledge by communicating with all the people which they cannot do if they studying by the traditional way of learning that is the classroom learning. Mobile learning is not the learning which can only be done through the internet or any of the mobile networks which connect the people around the world with each other. The mobile leaning also includes the applications which can be installed on the mobile devices which is originally develop for the education purpose we can say that these applications are the applications which when installed provide us the study material according to the course for which we have bough that application. This type of learning is known as the offline mobile learning where there is no need for the internet the student can read the study material that is packed inside the application. Mobile learning is becoming more and more popular because the mobile learning uses all the new advancement in the mobile phones. For e.g. the advancement of the $3 \mathrm{G}$ technology in the mobile devices allows the mobile learning to use this technology as well. With the introduction of the $3 \mathrm{G}$ in the mobile learning it allows the users to do video communication with each other which really boost the mobile learning. The next advancement in the field of the mobile devices which boost the mobile learning to its greater extent is the use of the automated speech recognition. It allows the users from the different regions to communicate with the each other through voice. For e.g. the two persons are communicating with each other using the mobile devices one is the person from the China and the other person is from India. By using the speech to speech recognition the person from china will communicate from his own language and the speech to speech recognition will automatically convert the communication from the Chinese language to the language which is understandable to the person in India.

But if dumb people want to communicate with other people through mobile devices they currently have only one way that is text based communication i.e. the dumb people has to write the text in their mobile in order to discuss their problems or give suggestions to the others. This method of communication is time consuming depending upon the typing skills of the particular one and moreover it requires the dumb people to type every time when they want to discuss something. In order to improve the communication method and increase the number of users of mobile learning an automated hand gesture recognition system for mobile learning can be used.

As the mobile learning research is still in its growing stage, and the research in the field of mobile learning is small as compare to the research in the field of e-learning. The prior literature review in the field of mobile learning includes how 
to embed the mobile learning with the new technologies, issues in the field of mobile learning, focus on design, developing the learning strategy for the mobile learning and how the mobile learning differs in heavy and light mobile phones. Yet there is a lot of researches still have to happen in the field of the mobile learning, none of the research in the field of the mobile learning is focused on how the mobile learning is useful for establishing communication of dumb people with the other people.

\section{HAND GESTURE RECOGNITION}

The process of capturing the hand movements is known as the hand gesture recognition. In this process the sequence of hand motions are captured by the camera then these sequence of hand motions are then segmented from the rest of the body parts and also from the background elements after isolating the hand gestures from the rest of the objects next step is to develop some type of grammar which will act as the instruction for the particular hand gesture and perform the required task.

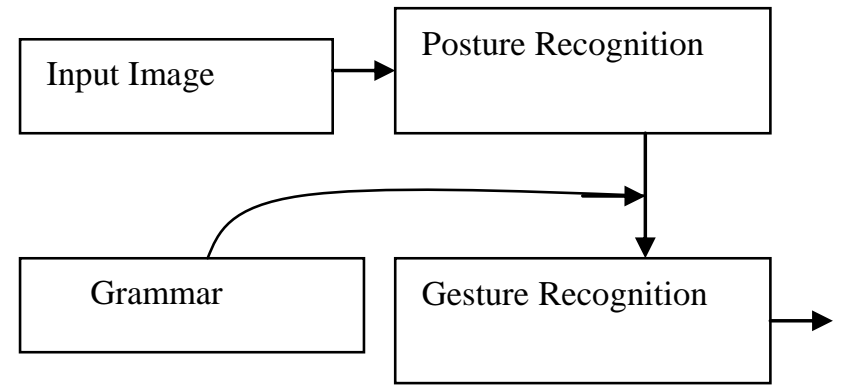

Figure: Process of hand gesture recognition [10]

\section{APPROACH FOR DEVELOPING THE PROPOSED SYSTEM}

The proposed system based on the research that is discussed so far will firstly capture the hand sign/movement of the dumb people using the webcam. This can be done by doing programming in asp.net with vc++ and opencv which can be embedded easily in asp.net with vc++. To capture the hand sign of dumb people cvCaptureFromCam function will be used in our programming language environment (which is asp.net with vc++ and opencv). Now after capturing the hand sign of the dumb people next step is to do the image preprocessing.

The preprocessing of image includes the conversion of color space and image resizing. The conversion of color space is performed right after video is captured by the webcam. Under this step we convert the color space RGB to HSV. To convert RGB to HSV cvCVTColor function is used which has three arguments first one is the source image second one is the destination image and the third argument is to define the pattern in which we have to change the video in this case this will be CV_BGR2HSV. Now after getting the video in the HSV now it is necessary to define the skin color range according to the HSV video. For defining the skin color range the cvScalar function of opencv is used which has 4 arguments which will define the color value in integer number. Now after defining the skin color range now in the video the part of the video which comes in the skin color range replace it with white pixels and replace the rest of the image with black pixels this is shown in figure 3. Then after preprocessing the next step is segmentation. In this step the area of interest from the video gets separated from the rest of the video in this we want only the hand as the area of interest in the video so we draw a rectangle in the area of the hand by using the cvRectangle function of opencv. After the image is being segmented then The proposed system use the image morphology algorithm to omit the noise from the image that results after segmentation. The noise means that while defining the skin color range of the system it may involves the background color that lies in the skin color range so the process of making this pixel black one is known as the omitting noise from the image. To find center of the captured gesture of the hand is needed to find out how many fingers are open or close using convex hull algorithm.

After the segmentation we can find the centre of the hand by getting the coordinates of the rectangle in which our hand is present then after getting the coordinates we can easily find out the center of our hand and after knowing the center of the hand we can compute the hand size by computing the radius of the palm .Now to obtain the size of the hand firstly we draw a circle on the center of the hand and we increase its radius until we find the first black pixel (because the hand lies in skin color range so all the pixels of hand region will be white and rest of the video pixels will be black). So after drawing the circle with increasing its radius until it meets the first black pixel then the length from the center which will compute is the radius of the hand[2]. That's why the image segmentation is important part because if due to the background a black pixel occurs before the circle will be smaller than it actually should be.

The proposed system will use the algorithm which will use to find out the number of visible fingers in the image named as the convex hull algorithm. In this algorithm firstly the center of the hand is find out. Because this algorithm is based on the center of the hand after finding the center of the hand it will then check how many fingers are visible which means whether there is any finger which is folded or not .To achieve it what we actually do is firstly we find out the radius of the hand with all the fingers closed and after this check the distance from the center of the hand to the point where first black pixel meets. If the distance is same as that of its radius means the finger is closed and if the distance is larger than its radius it means the finger is open by using it we can find how many fingers are open or close. After checking how many fingers are visible the next step is to store the video in the database. The video can be stored to database either in the binary form or with the pattern of any length generated by the number of visible fingers (for e.g. if we decided to store the pattern of length 4 then if the first frame of the video contain the one open finger and second frame contain the 2 open fingers third contain the 3 open fingers and fourth contain the 4 open fingers then the pattern will be 1234) along with the text that will be correspond to its respective video. When the user give its hand moment as the input in the form of video then the video will go through all above mentioned steps and 
then match its value in the database values of video if the match occurs it will send the corresponding text to the user which makes the communication between the dumb people and other people possible in mobile learning. Now when the user will use this system to communicate with the other people in the network through the mobile devices then the user will use the webcam to capture the hand sign and this hand sign will be converted in the format in which all the images stored in the database after the hand sign is converted in that format then the captured hand sign will be match with all the database values and when ever a match is occur in the database it will send the corresponding text to the person to whom the person is communicating. The proposed system can implement in the asp.net technology which have the opencv library. The process of the whole system is shown in figure 1 and the flow of data of the system is shown in figure 2 . The proposed system will store the video in the form of the sequence of the number of fingers opened by capturing the output given by the convex hull algorithm. Storing the images with the sequence of the number of visible fingers have so many advantages if we use the binary format to store the image it will need to store the same images with different spaces between the fingers which will be overhead for the user as well as the erroneous in system will also increase.

\subsection{Figures and Tables}

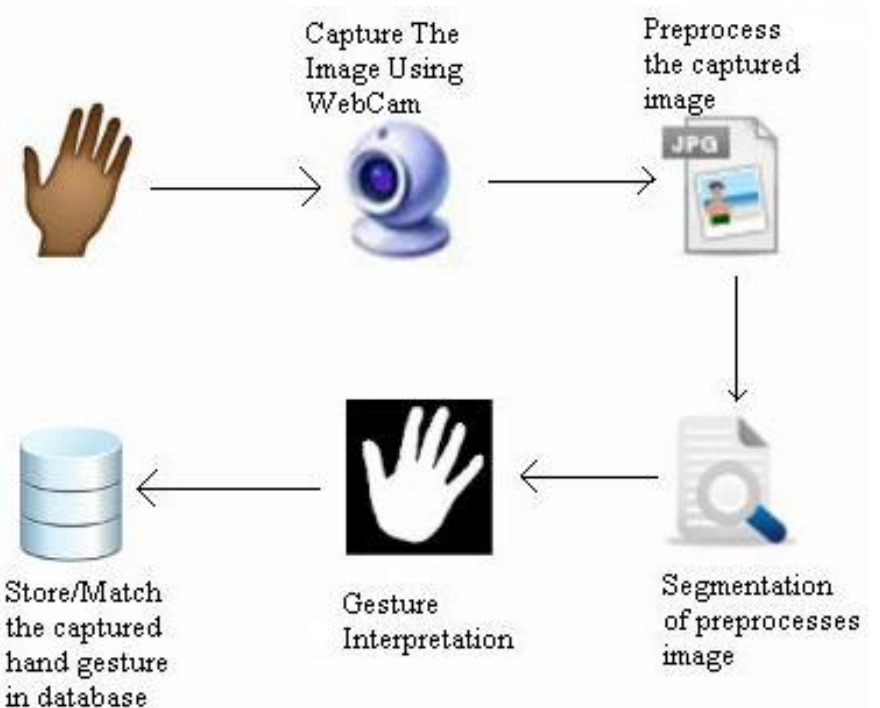

Figure 1: The block diagram of proposed system

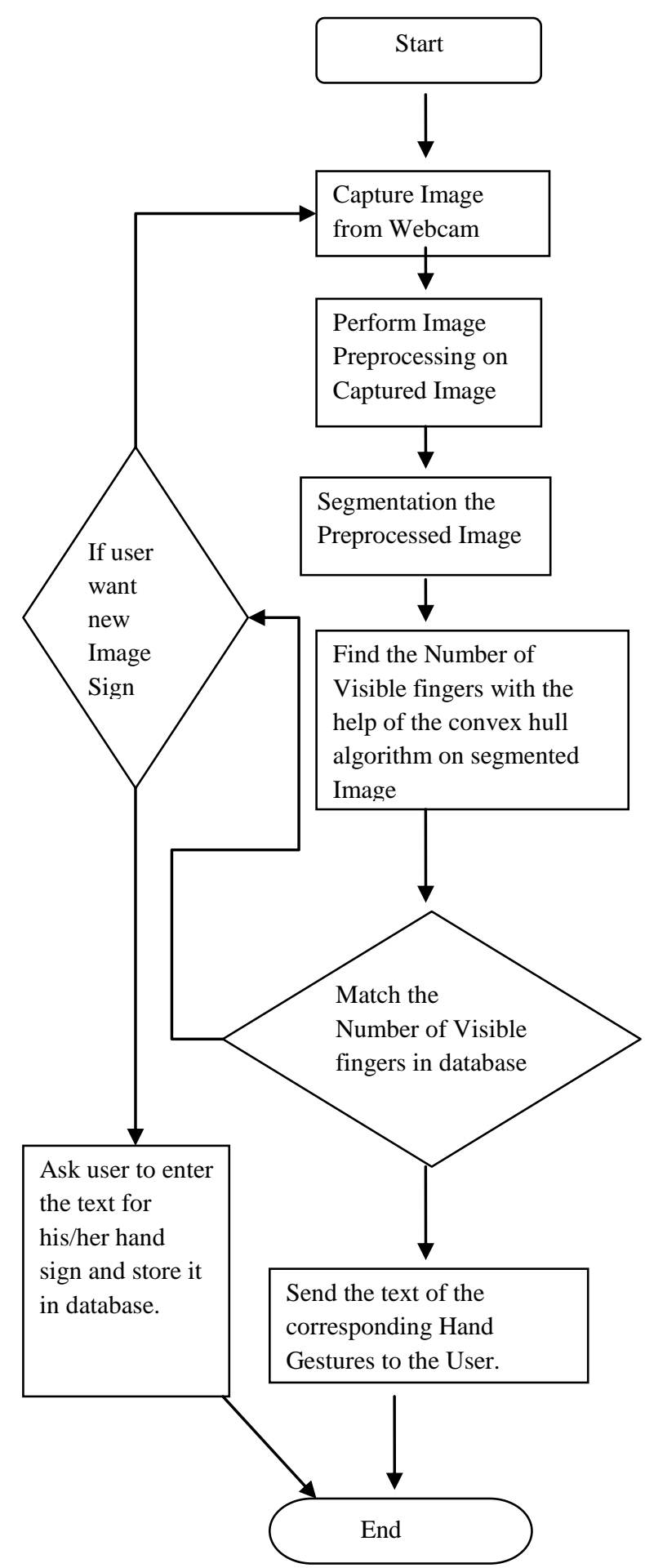

Figure 2: Flow Chart of the Proposes System. 


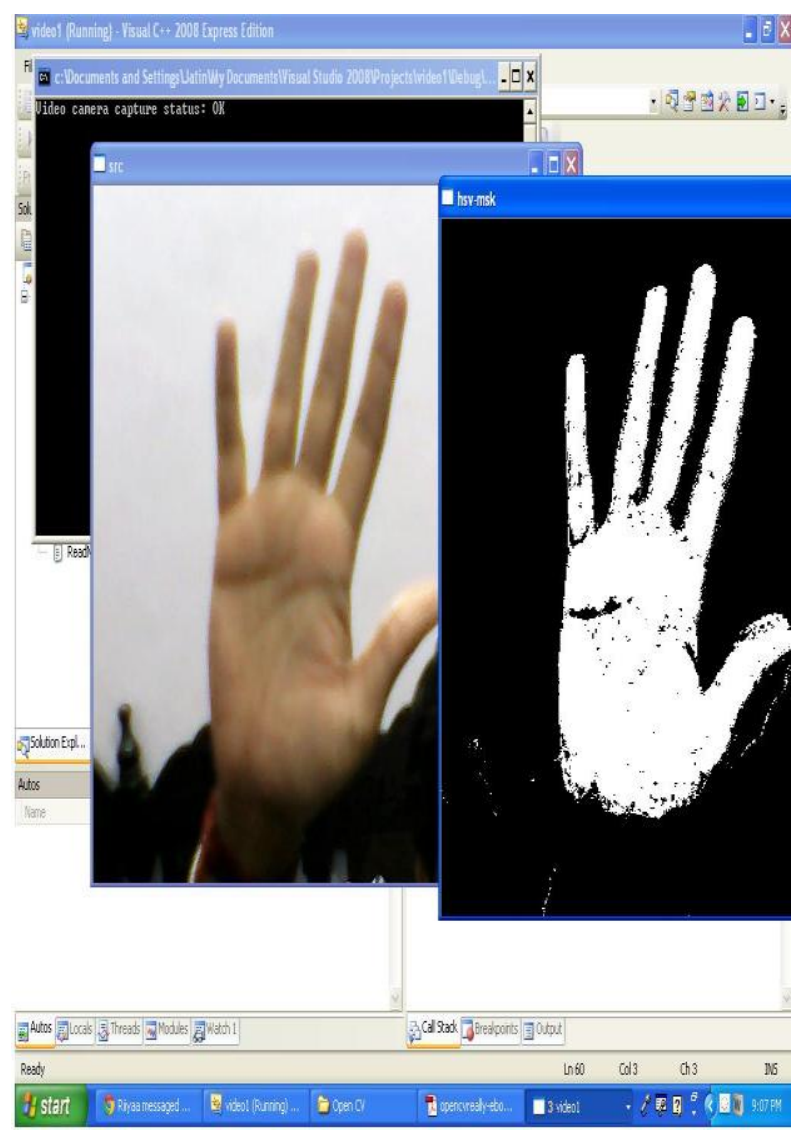

Figure 3: Converting the skin color range into white pixels and rest of the background to black pixels.

\section{PURPOSE OF THE RESEARCH}

The purpose of this research is to develop a system which can be used for the dumb people. The proposed system will takes the input from the hand movements of the dumb people and using their hand moments they can communicate with the other people. The highlighting goals of this research are:-

1) To develop such a system, this allows the dumb people to use their hand sign language to interact with others.

2) A system which allows its users who are dumb to communicate with other people without typing the text from the mobile device.

\section{BENEFITS OF THE RESEARCH}

This research will increase the number of users in the field of mobile learning. Till now most of the research in the mobile learning field gives much of the benefits to the people who can speak. But the people who cannot speak are unable to exploit all the benefits of the research in mobile learning as it is exploited by the people who can speak e.g. the implementation of the voice communication in mobile learning. This research will allow the dumb people to actively participate in the learning activities; they can share their idea and discuss with other people that are connected in the network (through internet). By giving the suggestions dumb people can make the people who can speak to understand much more about some topics and these people can learn a lot from the dumb people and also get to know the ideas of dumb people. This research will allow people who cannot speak to use all the features of the mobile learning and explore the content of the education as much they can either by studying or by discussing it with others. With the help of this research the dumb people can let the people who can speak know about what they want to say to them. By getting the education, the dumb people can contribute a lot for the country which will help in the development for the country. This will significantly increase the educational rate of the country. The main significance of the purposed system based on the above research is to provide the education to the dumb people on the same platform and environment in which the other people will study so that the dumb people can also take part effectively in the learning process. Making the dumb people educated will allow them to share their knowledge with others which can be helpful for the other persons because the learning resources are the same for the dumb people as well as for the people who can speak so when the dumb people can get more knowledge about the topics than other people. So the proposed system will contribute a lot in the field of Mobile Learning. This Proposed system will help dumb people in addition to it this system will prove beneficial for general people as it remove awkward feeling that typing on keyboard. This system can be used anywhere without need of any sort of supporting files and platform.

\section{CONCLUSION}

The proposed system will allow the dumb people to do communication and discuss the problem with the other people which will increase the number of mobile learning users and also by educating the dumb people will allow the education rate of a country to be increase and by using this system the dumb people can share their ideas with the other people.

The limitation of this proposed system includes that the system will take the static input of the hand movements of the user and will do the image processing on in and apply the convex hull algorithm on it. And will send the text to the other people. The text is also statically stored in database corresponding to the hand movements that matches it can be send in text or in speech with the help of text to speech converter.

The applications of the proposed system is the development of the country that having all the people either they are dumb or the normal people to be educated and can develop a country which have low unemployment rate without going to the colleges or schools.

\section{ACKNOWLEDGMENTS}

We will like to thank our advisor Mr. Sawal Tandon for guiding us all the time in our project and also all our classmates who helps us when we need their help and also all the fellow professors for supporting us all the time and providing us each and every possible help they can provide to us. Beside the entire professor team and our advisor we like to thank our thesis evaluator team who gave us their valuable suggestions to implement that in our project. And last but not the least we like to thank our parents firstly because they gave birth to us and also supporting us throughout our life in each and every situation.

\section{REFERENCES}

[1] Ayman Atia, Shin Takahashi, Kazuo Misue, and Jiro Tanaka,2009. UbiGesture: Customizing and Profiling Hand Gestures in Ubiquitous Environment , J.A. Jacko (Ed.): Human-Computer Interaction, Part II, HCII 2009, LNCS 5611, pp. 141-150, 2009.

[2] Hojoon Park ,2008. A Method For Controlling The Mouse Movement using a Real Time Camera, Brown University ,Providence ,RI ,USA, Department of computer science.

[3] Paul Pocatilu, Adrian Pocovnicu,2010. Mobile learning Application Audit, 2010 informatica Economica vol 14, No 1. 
[4] Qing Chen ,2007. A Basic introduction to opencv using Image Processing, School of Information Technology and engineering ,University of Ottawa.

[5] Shao Chun Li, KO Kang Chun, 2011.Apply Problem Based Learning in Mobile Learning Environment, 2011 11th IEEE International Conference on Advance Learning Technology.

[6] SCOE Information Technology,2011.Controlling computer using hand gesture recognition, Chapter 2, Requirement Gathering.

[7] Yazen Zhang, Jina Li, 2011.Application of 3G based mobile learning in teacher training, 2011 Fourth International Conference on Information and Computing.
[8] Yuan Jiugen, Xing Ruonam, Wang Jianmin, 2010.Applying Research of Mobile Learning Mode in Teaching, 2010 International Forum on Information Technology and Application.

[9] Yikai Fang, Kongqiao Wang, Jian Cheng, Hanqing Lu,2007. A Real Time Hand Gesture recognition Method, 2007 11th international conference.

[10] Pragti Garg, Naveen Aggarwal and Sanjeev Sofat 2009, Vision based Hand Gesture Recognition, World Academy of Science, Engineering and Technology 492009 